\title{
Ramos-Arroyo syndrome
}

INSERM

\section{Source}

INSERM. (1999). Orphanet: an online rare disease and orphan drug data base. Ramos-

Arroyo syndrome. ORPHA:1051

Ramos-Arroyo syndrome (RAS) is a very rare genetic disorder characterized by corneal anesthesia, retinal abnormalities, bilateral hearing loss, distinct facies, patent ductus arteriosus, Hirschsprung disease (see these terms), short stature, and intellectual disability. 